\title{
Sex-Based Differences in Smgc Expression in the Submandibular Gland of C57BL/6 Mice
}

\author{
Yuko Kusakabe $^{\text {a }}$ Yumiko Shindo $^{\text {a }}$ Takayuki Kawai $^{\text {a }}$ Yoko Takahashi $^{\mathrm{a}}$ \\ Masuko Kobori $^{a}$ Hiroko Inoue $^{b}$ Ichiro Saito $^{c}$ \\ a Division of Food Function Research, Food Research Institute, NARO, Tsukuba, b Department of Pharmacotherapy, \\ Nihon Pharmaceutical University, Ina, and 'Department of Pathology, Tsurumi University School of \\ Dental Medicine, Yokohama, Japan
}

\section{Key Words}

Sex-based differences · Salivary proteins $\cdot$ Estrogen .

Ovariectomy $\cdot$ Menopause submandibular gland. Our findings suggest that salivary proteins have potential as markers for evaluating therapies for menopausal symptoms.

\begin{abstract}
Objective: The decrease in female hormone levels at menopause affects whole-body homeostasis. Various therapies including hormone therapy and treatment with herbal supplements are available to improve menopausal symptoms. However, a method for evaluating their effectiveness has not been established. We sought to identify useful biomarkers to assess therapy efficacy. Methods: We searched for salivary proteins affected by changes in female hormone levels in mouse submandibular glands. Results: The expression of submandibular gland protein C (Smgc) was decreased following ovariectomy, while the expression of the alternative splicing transcript $t$-Smgc was increased. Notably, Smgc expression increased following $\beta$-estradiol administration, and was barely detectable in the submandibular glands of male mice. Conclusion: The results suggest that Smgc expression may be estrogen dependent. Moreover, changes in the SMGC protein amount in the saliva were in accordance with those in mRNA expression in the
\end{abstract}

\section{Introduction}

Female hormones such as estrogen and progesterone are important for menstruation and maintaining homeostasis. Estrogen production dramatically decreases at menopause, causing symptoms including hot flushes, night sweats, dry skin and mucosa, and osteoporosis. Hormone therapy is a well-established treatment for menopausal symptoms; however, adverse effects of female hormones have limited their widespread use. Alternative treatments such as herbal supplements are available; however, there is no useful method to evaluate their effectiveness.

Biomarkers are important tools for evaluating medical therapies. Molecules with activity regulated by certain therapies are especially useful for assessing therapy efficacy; for example, various molecules have been identified as biomarkers for cancer treatments $[1,2]$.

\section{KARGER}

E-Mail karger@karger.com www.karger.com/pat
(C) 2016 S. Karger AG, Basel

$1015-2008 / 16 / 0836-0287 \$ 39.50 / 0$
Yuko Kusakabe

Division of Food Function Research, Food Research Institute, NARO

2-1-12 Kannondai, Tsukuba, Ibaraki 305-8642 (Japan)

E-Mail ykusa@affrc.go.jp 
Table 1. Primer sets for RT-PCR

\begin{tabular}{|c|c|c|}
\hline Gene & Primer sequence & Gene bank accession No. \\
\hline$S m g c$ & $\begin{array}{l}5^{\prime}-\text { ACAGTCTCTACACTTAGGTCCCA-3' }(+9 \text { to }+23) \\
5^{\prime} \text {-ACTTGTGATCGGTTCGACTATT-3' }(+716 \text { to }+695)\end{array}$ & NM_198927 \\
\hline$t-S m g c$ & $\begin{array}{l}5^{\prime} \text {-ACAGTCTCTACACTTAGGTCCCA- } 3^{\prime}(+9 \text { to }+23) \\
5^{\prime} \text {-GGATGACCAGTCACAAACACTA-3' }(+2,646 \text { to }+2,625)\end{array}$ & NM_198927 \\
\hline Muc19 & $\begin{array}{l}5^{\prime} \text {-GATTATGCGATTGGTTCATCCT-3' }(+22,175 \text { to }+22,196) \\
5^{\prime} \text {-GTGCAATGTCCCTGAACTCATA-3' }(+22,523 \text { to }+22,502)\end{array}$ & NM_207243 \\
\hline$G P D H$ & $\begin{array}{l}5^{\prime}-\text { ATGGCGTTTCAAAAGGCAGT-3' }(+320 \text { to }+339) \\
5^{\prime} \text {-AATCCTCCACAACTACGGTC- } 3^{\prime}(+2,499 \text { to }+2,480)\end{array}$ & NM_001145820 \\
\hline
\end{tabular}

Estrogen regulates the expression of multiple target genes known as estrogen-responsive genes, including those encoding efp (estrogen-responsive finger protein) [3], prolactin [4], pS2 [5] and uteroglobin [6]. Estrogen binds to estrogen receptors (ERs), which are members of the nuclear receptor superfamily and act as ligand-dependent transcription factors to regulate target gene expression by directly binding to specific DNA sequences called estrogen response elements (EREs) [7]. Incomplete EREs or ERE half-sites are regulated by other transcription factors, including AP-1 (activating protein 1) [8], Sp1 (specificity protein 1) [9] and NF- $\kappa B$ (nuclear factor- $\kappa B$ ) [10]. Most estrogen-responsive genes have been found in the brain [11], bone marrow [12], heart [13] and other tissues. These organs can only be accessed by invasive approaches, preventing the development of a method to easily evaluate the effectiveness of menopause treatments.

A potential noninvasive approach for the evaluation of hormone therapy is the use of salivary samples. Salivary glands exhibit sexual dimorphism; volumes of the parotid and submandibular glands are reportedly larger in males, as is the saliva flow rate [14]. Dry mouth often occurs in women experiencing menopause [15], and most sex hormones are present in saliva at levels that correlate with those in blood $[16,17]$. Moreover, salivary glands are reported to express ERs $[18,19]$. Unfortunately, there is a dearth of information regarding the specific molecules regulated by estrogen and ERs in the salivary system.

Here, we attempted to identify estrogen-responsive genes in the mouse submandibular glands and saliva. This led to the identification of the submandibular gland protein $\mathrm{C}$ gene $(\mathrm{S} m g c)$ as an estrogen-responsive gene that could be used to evaluate female hormone therapy and its alternatives in mice.

\section{Materials and Methods}

\section{Experimental Animals}

Seven-week-old male and female C57BL/6J mice were purchased from Charles River Laboratories Japan (Yokohama, Japan) and fed modified AIN-93G containing corn oil instead of soybean oil to avoid isoflavones, which are phytoestrogens. All experimental procedures were approved by the Institutional Animal Care and Use Committee (IACUC) at the National Food Research Institute (NARO; approval No. H24-009, H25-014, H26-019). The mice were killed by cervical dislocation, and their submandibular glands were extirpated and used for preparing RNA for reverse transcription-polymerase chain reaction (RT-PCR) and for preparing protein samples. The extirpations were carried out in the laboratory registered with the NARO IACUC.

\section{Ovariectomy and Subsequent Estrogen Therapy}

Female mice were sham-treated or ovariectomized (OVX) under isoflurane anesthesia. For one experiment, 12 OVX mice were divided into 2 groups: the first group was treated with daily subcutaneous $17 \beta$-estradiol injections $\left(0.6 \mu \mathrm{g} \cdot \mathrm{kg}^{-1} \cdot \mathrm{day}^{-1}\right.$; Sigma-Aldrich, St. Louis, Mo., USA), beginning 1 week after OVX, and the other group served as a control. $17 \beta$-estradiol was dissolved in DMSO at $2.5 \mu \mathrm{g} / \mathrm{ml}$ and mixed with phosphate-buffered saline before use.

\section{Saliva Collection}

Three weeks after surgery, the mice were weighed and anesthetized with an intraperitoneal injection of pentobarbital $(36 \mathrm{mg} / \mathrm{kg})$. Pilocarpine $(0.1 \mathrm{mg} / \mathrm{kg})$ was intraperitoneally injected to stimulate salivation, and saliva secreted into the oral cavity was collected by capillary action using disposable glass capillary pipettes (Hirschmann Laborgeräte GmbH \& Co. KG, Eberstadt, Germany).

\section{RNA Extraction and cDNA Microarray Analysis}

Total RNA was extracted from the submandibular gland using the illustra RNAspin kit (GE Healthcare UK Ltd., Amersham, UK) and used for cDNA microarray analysis and RT-PCR. First-strand cDNA was reverse transcribed from $500 \mathrm{ng}$ total RNA from the submandibular glands using SuperScript III Reverse Transcriptase (Life Technologies, Carlsbad, Calif., USA). The microarray analysis was performed with a GeneChip ${ }^{\circledR} 3^{\prime}$ IVT Express Kit (Affymetrix Japan KK, Tokyo, Japan) as reported previously [20]. 


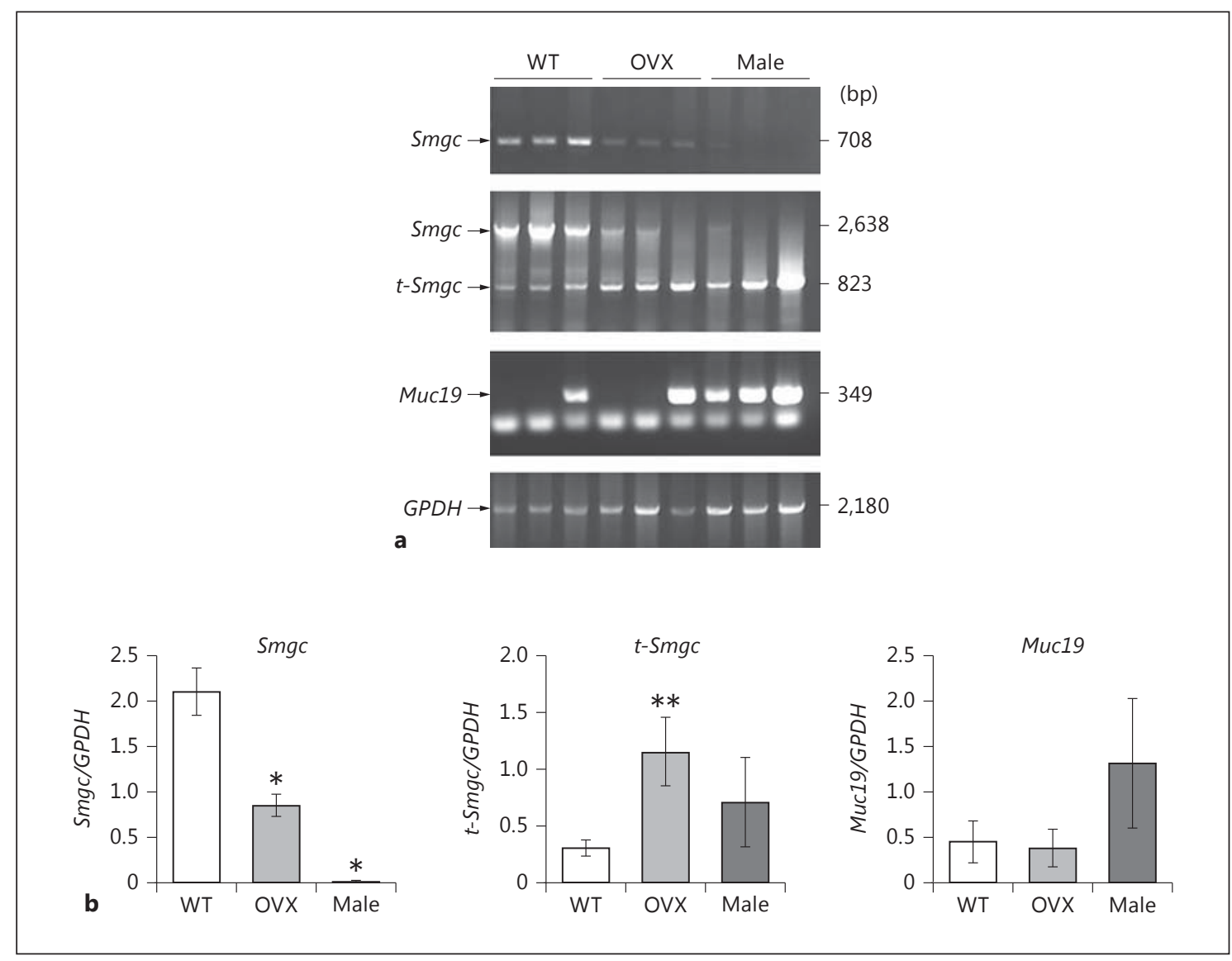

Fig. 1. Expression of the $S m g c / M u c 19$ gene in mouse submandibular glands. mRNA levels of $S m g c, t-S m g c$ and $M u c 19$ were quantified by semiquantitative RT-PCR. a Representative results of $S m g c$, $t-S m g c$ and $M u c 19$ mRNA in the submandibular glands of shamtreated mice (WT), OVX mice and male mice. GPDH served as the

\section{$R T-P C R$}

The primers were synthesized based on published sequences (table 1). RT-PCR was performed using $25 \mathrm{ng}$ of total RNA, $10 \mu \mathrm{M}$ of each primer, and rTaq DNA polymerase (TaKaRa Bio, Otsu, Japan). After $3 \mathrm{~min}$ at $95^{\circ} \mathrm{C}, 20-40$ cycles of $95^{\circ} \mathrm{C}$ for $1 \mathrm{~min}, 55^{\circ} \mathrm{C}$ for $1 \mathrm{~min}$, and $72^{\circ} \mathrm{C}$ for $1 \mathrm{~min}$ were performed, followed by a final extension at $72^{\circ} \mathrm{C}$ for $7 \mathrm{~min}$. Amplified sequences were visualized by gel electrophoresis on a $0.8 \%$ agarose gel and quantified using the CS Analyzer software (ATTO, Tokyo, Japan).

\section{Western Blot Analysis}

Frozen submandibular glands were homogenized and lysed using CelLytic MT (Sigma-Aldrich). Saliva samples were directly mixed with NuPAGE LDS sample buffer (Life Technologies). Sodium dodecyl sulfate-polyacrylamide gel electrophoresis (SDSPAGE) and Western blot analyses were performed as described previously [21]. A polyclonal antibody for SMGC (Everest Biotech Ltd., Upper Heyford, UK) was used at a dilution of 1:1,000 for saliva samples and of 1:5,000 for salivary gland samples, which were subsequently incubated with horseradish peroxidase-linked anti- internal control. b The bar graph shows the average relative amplicon band intensity as determined by densitometry $(n=6-25)$. ${ }^{*} \mathrm{p}<0.001,{ }^{* *} \mathrm{p}<0.01$ compared with sham-treated mice (one-way ANOVA followed by Dunnett's test). Values are expressed as means \pm SEs.

goat IgG (1:2,500; GE Healthcare UK). The signals were observed with LightCapture (ATTO) and quantitation was carried out using the CS Analyzer software.

\section{Plasmid Construction}

The different regions of the $S m g c$ promoter were cloned into the luciferase reporter vector pNL1.1 (Promega, Madison, Wis., USA). XETL [22] subcloned into the pNL1.1 vector was used as a positive control.

\section{Luciferase Assay}

The HEK 293 cell line (Life Technologies) was cultured in Dulbecco's modified Eagle's medium (Sigma-Aldrich) supplemented with $10 \%$ fetal calf serum (Life Technologies) at $37^{\circ} \mathrm{C}$ in a $5 \% \mathrm{CO}_{2}$ humidified incubator. For all experiments, $1.67 \mathrm{ng} / \mu \mathrm{l}$ plasmid DNA was used for transfection. The reporter plasmids were transfected using Lipofectamine LTX (reagent:DNA, 3:1; Life Technologies). $17 \beta$-estradiol $(100 \mathrm{nM})$ was added $24 \mathrm{~h}$ after the transfection. The luciferase assay was carried out after $24 \mathrm{~h}$ of $17 \beta$-estradiol treatment using the Nano-Glo-Luciferase Reporter Assay system (Promega). 


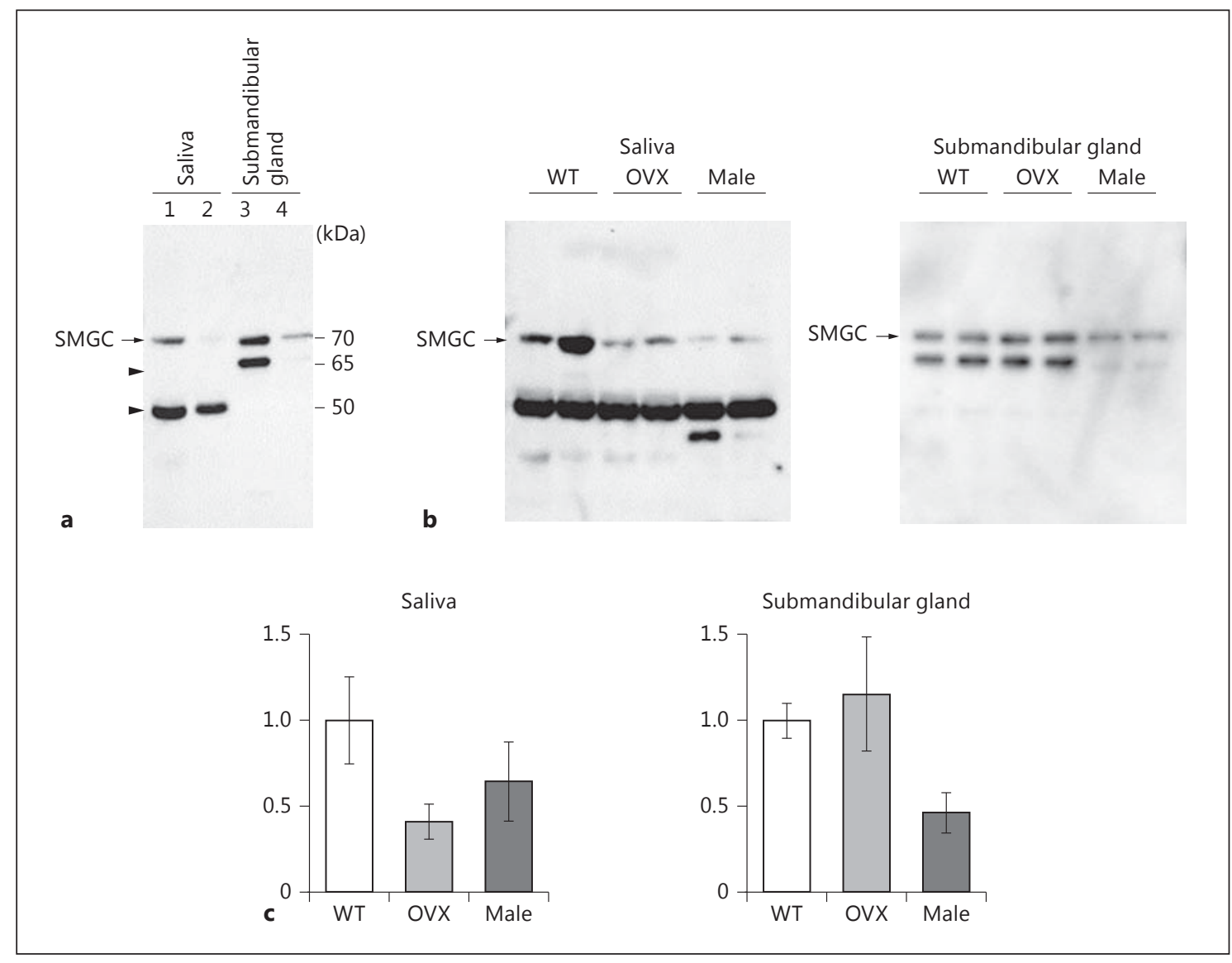

Fig. 2. SMGC protein expression in saliva and the submandibular gland. a Representative Western blot of SMGC expression in saliva and the submandibular gland. The arrow and arrowheads indicate SMGC protein and nonspecific bands, respectively. Total protein (lane 1, $24 \mu \mathrm{g}$ from saliva; lane 2, $8 \mu \mathrm{g}$ from saliva; lane 3, $4.5 \mu \mathrm{g}$ from the submandibular gland; lane 4, $0.9 \mu \mathrm{g}$ from the submandibular gland) was analyzed for each sample. b SMGC protein

\section{Statistical Analysis}

Quantitative RT-PCR, Western blot and luciferase assay data were compared with one-way analysis of variance (ANOVA) followed by Dunnett's test using the SigmaPlot software (Systat Software Inc., San Jose, Calif., USA). p $<0.05$ was considered significant.

\section{Results}

\section{Identification of Estrogen-Responsive Genes in the Submandibular Glands}

To identify proteins with an estrogen concentrationdependent expression, we compared gene expressions in the submandibular glands of OVX and sham-treated C57BL/6J mice using cDNA microarray technology. We expression in saliva and the submandibular glands of sham-treated mice (WT), OVX mice and male mice. Total protein from saliva $(24 \mu \mathrm{g})$ and the submandibular gland $(4.5 \mu \mathrm{g})$ were analyzed. The arrows indicate SMGC protein. c Relative protein levels of SMGC in saliva and the submandibular gland $(n=6-9)$. The average WT band intensity was normalized to 1 . Values are expressed as means \pm SEs.

selected the genes of which the average expression differed by more than 1.8-fold between the sham and OVX groups, annotated the selected 85 genes, and found the gene for SMGC to be highly expressed in the submandibular glands.

\section{Smgc Expression Is Estrogen Dependent in the}

Submandibular Glands

SMGC protein is a product of the $S m g c / M u c 19$ gene that encodes three transcripts: $S m g c$, truncated $S m g c$ $(t-S m g c)$ and mucin 19 (Muc19) [23, 24]. Smgc is expressed in terminal tubule cells, a type of transient secretory cell, in the rat submandibular glands $[25,26] . M u c 19$ is a member of the mucin family [27]. Mucins are glycoproteins responsible for mucus viscosity and elasticity. Smgc/Muc19 
is composed of 60 exons: $S m g c$ is encoded by exons 1-18, Muc19 is encoded by exons 1 and 19-60 [26], and the truncated splice variant $t$-Smgc is encoded by exons 1,17 and 18. Smgc is reportedly expressed during embryonic and early postnatal stages in rats and mice [23, 25], whereas $t$-Smgc is expressed in both neonatal and adult salivary glands [28]. To verify that these transcripts are related to estrogen, we performed RT-PCR with RNA from submandibular glands excised from OVX and sham-treated female and male mice (fig. 1). RT-PCR revealed that $S m g c$ expression was significantly reduced by OVX and was minimal in the submandibular glands of male mice. We used a primer pair for $t-S m g c$ capable of detecting both $S m g c$ and $t$-Smgc expression. RT-PCR analysis with this primer pair showed that $t-S m g c$ and $S m g c$ expressions were significantly increased and decreased, respectively, in the submandibular glands of OVX mice. These results suggested that OVX may produce alternative splicing. The primer pair for $t-S m g c$ was not able to detect $S m g c$ in male mice. Muc19 expression was higher in male mice than in sham-treated and OVX female mice. No significant difference between WT and OVX female mice was noted, likely due to interindividual differences in Muc19 expression.

\section{SMGC Protein Is Expressed in Saliva and the}

\section{Submandibular Glands}

Next, we analyzed $S m g c$ expression at the protein level by Western blotting with samples from saliva and the submandibular glands (fig. 2). First, we determined the specificity of the anti-SMGC antibody using samples from sham-treated female C57BL/6J mice. A $70-\mathrm{kDa}$ band was detected in both sample types; extra bands were detected at $50 \mathrm{kDa}$ in saliva and at $65 \mathrm{kDa}$ in the submandibular glands (fig. 2a). Because the calculated size of SMGC is $74.4 \mathrm{kDa}$, we selected the $70-\mathrm{kDa}$ bands as the SMGC-specific bands. Expression of the SMGC protein was lower in male mice than in sham-treated female mice in both saliva and the submandibular glands (fig. 2b, c). SMGC protein expression in the saliva of OVX mice was lower than in that of sham-treated mice; SMGC levels were approximately equal in the submandibular glands of both groups. Western blot analysis did not reveal significant differences among the sample types.

\section{Smgc Expression Is Regulated by $\beta$-Estradiol in the \\ Submandibular Glands}

The RT-PCR and Western blot results suggested that $S m g c$ expression is regulated by estrogen. To confirm estrogen-dependent expression, RT-PCR and Western blot analyses were carried out for OVX mice subcutaneously

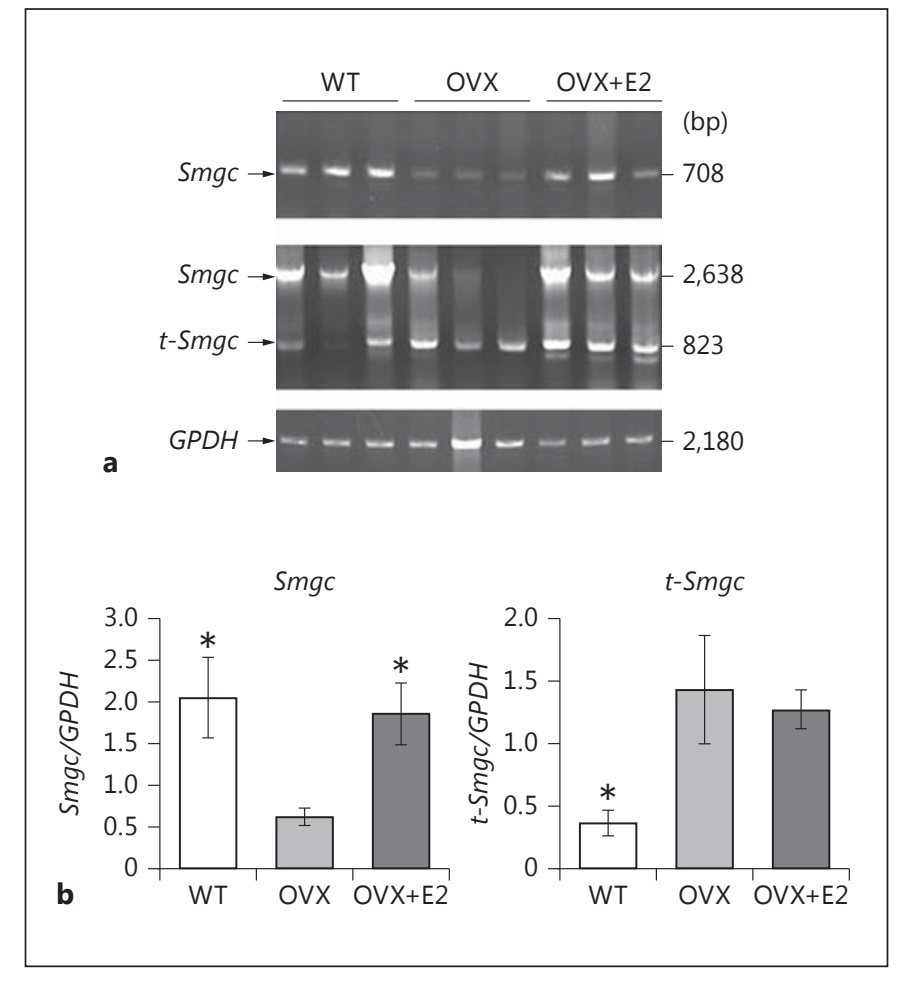

Fig. 3. Effect of estradiol on $S m g c$ mRNA expression in the submandibular glands of OVX mice. a Representative RT-PCR analysis results of $S m g c$ and $t-S m g c$ mRNA expression in the submandibular glands of sham-treated mice (WT), OVX mice and $\beta$ estradiol-treated OVX mice (OVX+E2). GPDH served as the internal control. $\mathbf{b}$ The bar graph shows the average relative amplicon band intensity determined by densitometry $(\mathrm{n}=9-12) .{ }^{*} \mathrm{p}<0.05$ compared with OVX mice (one-way ANOVA followed by Dunnett's test). Values are expressed as means \pm SEs.

injected with $17 \beta$-estradiol (fig. 3, 4). Smgc mRNA expression in the submandibular glands significantly increased with $17 \beta$-estradiol, while $t$-Smgc mRNA expression decreased slightly (fig. 3). The level of SMGC protein in both saliva and the submandibular gland also tended to increase, albeit insignificantly, with $17 \beta$-estradiol (fig. 4).

To evaluate whether the expression of Smgc and $t$ $S m g c$ is regulated by $\beta$-estradiol, we performed a sequence analysis of the $S m g c / M u c 19$ promoter region (5 kbp upstream of the transcription start site). As shown in figure 5a, the Muc19/Smgc promoter region contains five ERE half-sites. In the region $450 \mathrm{bp}$ upstream of the transcription start site, an ERE half-site, an AP-1 site and two SP1 sites were identified; however, this region lacks full EREs (fig. 5b). Luciferase assays of this promoter region showed that the promoter activity tended to decrease by deletion of the AP-1 site (fig. $5 \mathrm{c}$ ). 
Fig. 4. Effect of $\beta$-estradiol on SMGC protein expression in the submandibular glands of ovariectomized mice. a Representative results for SMGC protein expression in saliva and the submandibular glands of sham-treated mice (WT), OVX mice and $\beta$-estradiol-treated OVX mice $(\mathrm{OVX}+\mathrm{E} 2)$ assessed by Western blot analysis. Total protein from saliva $(24 \mu \mathrm{g})$ and the submandibular gland $(4.5 \mu \mathrm{g})$ were analyzed. b Relative protein levels of SMGC in saliva and the submandibular glands $(\mathrm{n}=4-9)$. The average WT band intensity was normalized to 1 . Values are expressed as means \pm SEs.

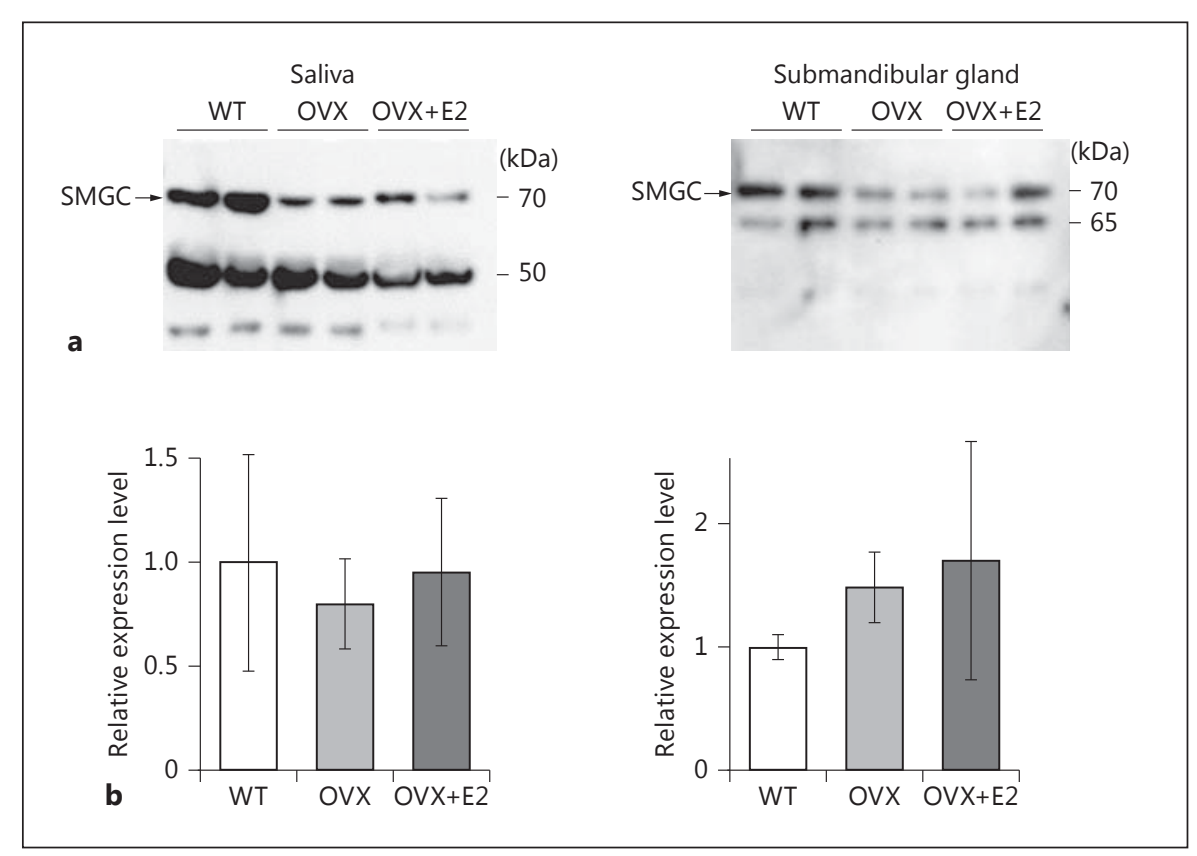

\section{Discussion}

In this study, we aimed to identify estrogen-responsive genes in the salivary system and found that Smgc expression was estrogen level dependent. In the process of annotating the selected genes, we found that cysteine-rich secretory protein 1 and demilune cell and parotid protein 3 genes are also expressed in the salivary glands. However, one-way ANOVA did not reveal any significant differences in gene expression, probably because the submandibular glands are comprised of heterogeneous cell populations.

Discrepant results have been reported for Smgc expression in adult mouse submandibular and sublingual glands. Zinzen et al. [23] showed that female but not male C57BL/6J adult mice express $\mathrm{Smgc}$ in the submandibular glands. Conversely, Culp et al. [24] reported that Smgc is expressed during the neonatal and early postnatal stages in the sublingual gland of NFS/NCr mice but not in the submandibular or sublingual glands of adult male and female mice. In the present study, we found that $S m g c$ expression is affected by estrogen in female C57BL/6J mice. The differences in these results can be attributed to the different genetic backgrounds used. Some NFS/N substrains have been reported to possess an abnormal salivary system. For example, the sublingual glands of a mutant substrain, NFS/sld, exhibit arrested differentiation in the sublingual glands [29]. NFS/sld mutant mice are used as an animal model for primary Sjögren's syndrome [30]. These studies suggest that the NFS strain may have a genetic predisposition to unusual salivary system development.

We found a clear inverse correlation between $S m g c$ and $t$-Smgc mRNA expression. In addition, we found an ERE half-site, AP-1 site and SP1 site within the 450-bp region upstream of the transcription start site. However, luciferase assays showed that the role of this region in the responsiveness to $\beta$-estradiol is very limited. This result suggests that female hormones might regulate Muc19/ $S m g c$ gene expression through as yet unknown regulatory systems. We did not analyze $S m g c$ and $t$-Smgc expression in developmental stages because high $S m g c$ expression has been reported during salivary gland development in both C57BL/6J and NFS/NCr mice [25, 28]. This high expression was observed irrespective of the sex and genetic background of the mice. Estradiol levels are higher in rat embryonic tissues than in newborn tissues [31], and plasma estradiol levels are high in both male and female neonatal rats, while the levels decrease with growth. Taken together, these findings suggest that estradiol likely drives high Smgc expression in the embryonic and neonatal salivary glands as it does in adult female mice.

We did not observe a coordinated expression of $S m g c$ and Muc19. Das et al. [28] showed that the expression level of $t$-Smgc correlates with that of Muc19. We found similar results in male mice, but OVX mice exhibited low Muc19 expression despite high $t$-Smgc expression. More- 
Fig. 5. Transcription activity of the $S m g c /$ Muc19 promoter region. a Schematic representation of the region upstream of the Smgc/Muc19 gene showing the locations of putative transcription factor binding sites. b Schematic representation of the region upstream of the $S m g c / M u c 19$ gene used for the reporter assay in c. c Luciferase assays. The luciferase reporter vectors are shown in the left panel. Quantitation of the luciferase expression is shown in the right panel. All the transfections were repeated more than 4 times, and the results are expressed as means \pm SDs. RLU = Relative light units; $\mathrm{tk}=$ thymidine kinase; Nluc $=$ NanoLuc luciferase; E2 = 17 $\beta$-estradiol.

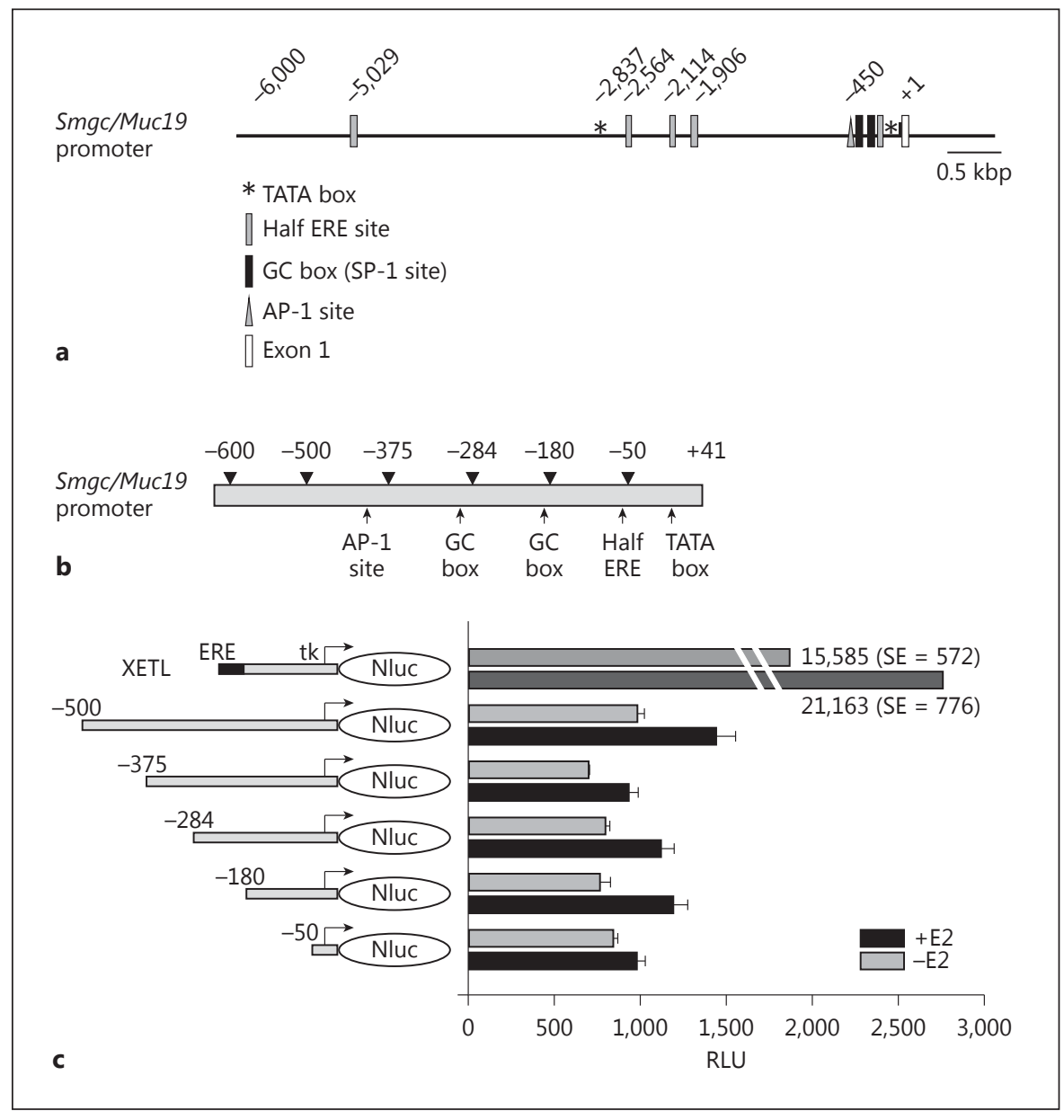

over, the individual differences in $M u c 19$ expression were high in our study. The inconsistent expression data could possibly be attributed to the PCR primer design. Additional studies are needed to clarify this issue.

Our study has several limitations. The most significant is that there is no human homologue of $S m g c$. Still, it may be a useful biomarker for mouse studies designed to assess menopause therapies. Furthermore, our microarray analysis did not reveal any gene clusters regulated by female hormones because individual differences were observed in the expression levels of most genes. If such clusters exist, there might be additional potential marker genes that are common to both mice and humans.

In our study, estrogen-based differences in SMGC expression between male and female mice were detectable at the protein level in saliva and the submandibular glands. We found a similar difference at the protein level in saliva between OVX and sham-treated female mice, but it was not significant. Moreover, the amount of
SMGC protein in the submandibular gland of OVX mice was higher than in sham-treated female mice, although not significantly. The SMGC protein level in the submandibular gland of sham-treated female mice is not necessarily higher than that in OVX mice because SMGC in the submandibular gland is constantly secreted into the oral cavity along with saliva, while Smgc mRNA is not. The anti-SMGC antibody used in our study had low sensitivity and specificity for quantitatively detecting SMGC protein, so further studies are needed to clarify its expression.

Estrogen regulates the expression of a wide array of genes and affects various organs. However, the effects of estrogen in most organs cannot be easily evaluated because it is difficult to sample tissues. Saliva is a biological sample that is relatively easily and painlessly obtained. Interestingly, estrogen-regulated gene expression reportedly occurs in the salivary gland [32]. In this study, we demonstrated estrogen-dependent SMGC protein 
expression in saliva samples and $S m g c$ expression in the mouse submandibular gland, with sex-dependent differences. Our results suggest the existence of femalespecific salivary proteins, the levels of which could be measured to evaluate the effects of estrogen or phytoestrogen.

\section{Acknowledgements}

We thank Ms. Masayo Okano for providing technical assistance. This work was supported by a grant for the research project on new demand creation of agricultural products of the Ministry of Agriculture, Forestry and Fisheries of Japan, and Fuji Foundation for Protein Research.

\section{References}

1 Kim WJ, Bae SC: Molecular biomarkers in urothelial bladder cancer. Cancer Sci 2008;99: 646-652.

2 Hassanein M, Callison JC, Callaway-Lane C, Aldrich MC, Grogan EL, Massion PP: The state of molecular biomarkers for the early detection of lung cancer. Cancer Prev Res (Phila) 2012;5:992-1006

3 Inoue $\mathrm{H}$, Ono K, Masuda W, Morimoto Y, Tanaka T, Yokota M, et al: Gender difference in unstimulated whole saliva flow rate and salivary gland sizes. Arch Oral Biol 2006;51: 1055-1060.

4 Maurer RA, Notides AC: Identification of an estrogen-responsive element from the $5^{\prime}$ flanking region of the rat prolactin gene. $\mathrm{Mol}$ Cell Biol 1987;7:4247-4254.

5 Berry M, Nunez AM, Chambon P: Estrogenresponsive element of the human $\mathrm{pS} 2$ gene is an imperfectly palindromic sequence. Proc Natl Acad Sci USA 1989;86:1218-1222.

6 Müller H, Beato M: RNA synthesis in rabbit endometrial nuclei. Hormonal regulation of transcription of the uteroglobin gene. Eur J Biochem 1980;112:235-241.

7 Klinge CM: Estrogen receptor interaction with estrogen response elements. Nucleic Acids Res 2001;29:2905-2919.

8 Webb P, Lopez GN, Uht RM, Kushner PJ: Tamoxifen activation of the estrogen receptor/AP-1 pathway: potential origin for the cell-specific estrogen-like effects of antiestrogens. Mol Endocrinol 1995;9:443-456.

9 Safe S: Transcriptional activation of genes by $17 \beta$-estradiol through estrogen receptor-Sp1 interactions. Vitam Horm 2001;62:231-252.

10 Harnish DC, Scicchitano MS, Adelman SJ, Lyttle CR, Karathanasis SK: The role of CBP in estrogen receptor cross-talk with nuclear factor- $\kappa \mathrm{B}$ in HepG2 cells. Endocrinology 2000;141:3403-3411.

11 Sakakibara M, Uenoyama Y, Minabe S, Watanabe Y, Deura C, Nakamura S, et al: Microarray analysis of perinatal-estrogen-induced changes in gene expression related to brain sexual differentiation in mice. PLoS One 2013;8:e79437.
12 Börjesson AE, Lagerquist MK, Windahl SH, Ohlsson C: The role of estrogen receptor $\alpha$ in the regulation of bone and growth plate cartilage. Cell Mol Life Sci 2013;70:4023-4037.

13 Murphy E, Steenbergen C: Estrogen regulation of protein expression and signaling pathways in the heart. Biol Sex Differ 2014;5:6.

14 Vining RF, McGinley RA: The measurement of hormones in saliva: possibilities and pitfalls. J Steroid Biochem 1987;27:81-94.

15 Gröschl M: Current status of salivary hormone analysis. Clin Chem 2008;54:17591769.

16 Inoue $\mathrm{H}$, Ono $\mathrm{K}$, Masuda $\mathrm{W}$, Morimoto $\mathrm{Y}$, Tanaka T, Yokota M, et al: Gender difference in unstimulated whole saliva flow rate and salivary gland sizes. Arch Oral Biol 2006;51: 1055-1060.

17 Suri V: Menopause and oral health. J Midlife Health 2014;5:115-120.

18 Leimola-Virtanen R, Salo T, Toikkanen S, Pulkkinen J, Syrjänen S: Expression of estrogen receptor (ER) in oral mucosa and salivary glands. Maturitas 2000;36:131-137.

19 Välimaa H, Savolainen S, Soukka T, Silvoniemi P, Mäkelä S, Kujari H, et al: Estrogen receptor- $\beta$ is the predominant estrogen receptor subtype in human oral epithelium and salivary glands. J Endocrinol 2004;180:55-62.

20 Nishide Y, Tadaishi M, Kobori M, Tousen Y, Kato M, Inada M, Miyaura C, Ishimi Y: Possible role of S-equol on bone loss via amelioration of inflammatory indices in ovariectomized mice. J Clin Biochem Nutr 2013;53: 41-48.

21 Shimizu M, Goto M, Kawai T, Yamashita A, Kusakabe Y: Distinct human and mouse membrane trafficking systems for sweet taste receptors T1r2 and T1r3. PLoS One 2014; 9:e100425.

22 Bunone G, Briand PA, Miksicek RJ, Picard D: Activation of the unliganded estrogen receptor by EGF involves the MAP kinase pathway and direct phosphorylation. EMBO J 1996:15: 2174-2183.
23 Zinzen KM, Hand AR, Yankova M, Ball WD, Mirels L: Molecular cloning and characterization of the neonatal rat and mouse submandibular gland protein SMGC. Gene 2004;334: 23-33.

24 Culp DJ, Latchney LR, Fallon MA, Denny PA, Denny PC, Couwenhoven RI, et al: The gene encoding mouse Muc19:cDNA, genomic organization and relationship to Smgc. Physiol Genomics 2004;19:303-318.

25 Ball WD, Hand AR, Moreira JE, Johnson AO: A secretory protein restricted to type I cells in neonatal rat submandibular glands. Dev Biol 1988;129:464-475.

26 Moreira JE, Hand AR, Ball WD: Localization of neonatal secretory proteins in different cell types of the rat submandibular gland from embryogenesis to adulthood. Dev Biol 1990; 139:370-382.

27 Bansil R, Turner BS: Mucin structure, aggregation, physiological functions and biomedical applications. Curr Opin Colloid Interface Sci 2006;11:164-170.

28 Das B, Cash MN, Hand AR, Shivazad A, Culp DJ: Expression of Muc19/Smgc gene products during murine sublingual gland development: cytodifferentiation and maturation of salivary mucous cells. J Histochem Cytochem 2009;57:383-396.

29 Hayashi Y, Kojima A, Hata M, Hirokawa K: A new mutation involving the sublingual gland in NFS/N mice: partially arrested mucous cell differentiation. Am J Pathol 1988;132:187191.

30 Haneji N, Hamano H, Yanagi K, Hayashi Y A new animal model for primary Sjögren's syndrome in NFS/sld mutant mice. J Immunol 1994;153:2769-2777.

31 Konkle AT, McCarthy MM: Developmental time course of estradiol, testosterone, and dihydrotestosterone levels in discrete regions of male and female rat brain. Endocrinology 2011;152:223-235.

32 Bretschneider N, Brand H, Miller N, Lowery AJ, Kerin MJ, Gannon F, et al: Estrogen induces repression of the breast cancer and salivary gland expression gene in an estrogen receptor $\boldsymbol{\alpha}$-dependent manner. Cancer Res 2008;68:106-114. 